\title{
Study of clinical profile of infantile tremor syndrome and its correlation with serum Vitamin B12 level
}

\author{
Jitendra Kumar Jain ${ }^{1}$, Gopikishan Sharma ${ }^{1}$, Dhaval Bhatt ${ }^{2}$, Amrit Lal Bairwa ${ }^{3}$, Ishwar Lal Meena ${ }^{2}$ \\ From ${ }^{1}$ Assistant Professor, ${ }^{2}$ PG Resident, ${ }^{3}$ Senior Professor and Head, Department of Pediatrics, Government Medical College, Kota, Rajasthan, India
} Correspondence to: Dr. Gopikishan Sharma, Department of Pediatrics, Government Medical College, Kota, 59 B Vijay Nagar, Baran Road Kota, Kota - 324 001, Rajasthan, India. E-mail: dr.gopikishansharma6162@gmail.com

Received - 03 May 2019

Initial Review - 25 May 2019

Accepted - 13 July 2019

\begin{abstract}
Objective: The objective of this study was to assess the clinical profile of infantile tremor syndrome (ITS) and to correlate serum Vitamin B12 level with ITS. Materials and Methods: This prospective study was done in the Department of Pediatrics of a Medical College of Kota. A total of 40 children, presenting with clinical features of ITS, were included in the study. A detailed history was taken. Investigations including complete blood count with blood indices (mean corpuscular volume, mean corpuscular hemoglobin $[\mathrm{MCH}]$, and $\mathrm{MCH}$ concentration), peripheral smear, and serum Vitamin B12 level were done in accordance with the WHO guidelines. Vitamin B12 deficiency was defined as serum Vitamin B12 concentration $<150 \mathrm{pmol} / \mathrm{L}$ or $203 \mathrm{pg} / \mathrm{mL}$. Results: All patients with ITS had pallor (100\%) and skin hyperpigmentation (100\%). Psychomotor changes in the form of apathy and/or developmental regression/stasis were found in $40(100 \%)$ cases. Regression of developmental milestones was found in $26(65 \%)$ and $10(25 \%)$ patients had stasis of milestones. Tremor was present in $14(35 \%)$ cases. Hepatomegaly was present in the majority $25(62.5 \%)$ of these children, whereas splenomegaly was present in only $8(20 \%)$ patients. Hypopigmented hair was found in only $12(30 \%)$ cases. Twenty-five $(62.5 \%)$ children were exclusively breastfed $(\mathrm{p}<0.05)$. Of the 40 cases, $26(65 \%)$ presented in prodromal phase and $14(35 \%)$ presented in the tremor phase. Among 26 patients of prodromal phase, $22(84.62 \%)$ had Vitamin B12 deficiency while among 14 cases of tremor phase, $9(64.28 \%)$ had Vitamin B12 deficiency. The majority of ITS children had macrocytic anemia (65\%) followed by dimorphic anemia (25\%). A significant number of ITS patients $(21,80.77 \%)$, having macrocytic anemia, had Vitamin B12 deficient. Conclusion: We conclude that children of ITS presented with anemia, skin hyperpigmentation, psychomotor changes, apathetic look, and developmental abnormality. The majority of children were on faulty feeding in the form of prolonged exclusive breastfeeding. Vitamin B12 deficiency was present in a significant number of patients with ITS.
\end{abstract}

Key words: Exclusive breastfeeding, Infantile tremor syndrome, Vitamin B12 deficiency

I nfantile tremor syndrome (ITS) is a clinical condition of infancy and early childhood characterized by acute or insidious onset with mental and psychomotor changes, pigmentary changes of hair and skin, pallor, and tremors [1,2]. It has been primarily reported from Southeast Asia including India and other developing countries. ITS is seen in infants and children of 6-36 months of age group [1], but majority of the cases reported under 6-18 months of age group [3,4]. Many theories have been proposed by different research workers regarding the etiopathogenesis of ITS, but the etiology of ITS is still elusive [5]. Various nutrient deficiencies (e.g., Vitamin B12, magnesium, zinc, Vitamin C, etc.) have been found to be associated with ITS. Other theories showed an association of viral encephalitis and degenerative processes with ITS $[1,6]$.

Among all proposed theories, the most widely accepted theory is nutritional theory or malnutrition theory. It is usually seen in children who are exclusively breastfed for prolonged periods by Vitamin B12 deficient vegetarian mother [6]. Vitamin B12 deficiency has been found to be associated with
ITS in many studies with normal serum ferritin and folic acid level $[1,5,7]$. The low levels of Vitamin B12 and its transport protein Transcobalamin II in the cerebrospinal fluid (CSF) may be responsible for the neurological features of this syndrome [8]. Zinc and magnesium deficiency have also been postulated to cause ITS. Iron and folic acid level were normal in some studies [5,7]. It is usual to find direct or indirect evidence of malnutrition associated other micronutrients deficiencies such as Vitamin A, D, and Vitamin B complex $[1,9,10]$.

Other factors for its etiology include viral encephalitis and degenerative processes. Seasonal incidence and cortical biopsy suggest that it might be due to meningoencephalitis. However, consistently normal CSF and failure to isolate any viral antigen do not support this hypothesis [6]. Metabolic or enzyme defect, a transient tyrosine metabolism defect, might lead to interference in melanin pigment production. Depigmentation of substantia nigra may explain tremors. Undeniable incidence of ITS is not known. In India, incidence is $0.2-2 \%$ of total pediatric hospital admissions $(1-2 \%$ in the $1960 \mathrm{~s}, 1.1 \%$ in $1975-77$, and $0.2 \%$ in 
the mid-1990s). Incidence rate of the disease was reduced over the years due to better weaning practices and improvement in nutritional status [11]. Therefore, this study was conducted to evaluate the clinical profile of ITS and to correlate the serum Vitamin B12 level with ITS.

\section{MATERIALS AND METHODS}

This was a one year, cross sectional study, conducted in Department of Pediatrics, Government Medical College and Associated Group of Hospitals, Kota, between December 2015 and November 2016. Children presented with pallor, skin hyperpigmentation, and psychomotor changes including apathy and/or developmental abnormalities were considered as prodromal stage of ITS and children presented with tremors with clinical features of prodromal stage were considered as tremor stage of ITS. Children presented with other comorbid neurological conditions, neurodegenerative disorder, preexisting cerebral palsy, mental retardation, developmental delay, postmeningitis, encephalitis, and/or meningoencephalitis sequel were excluded from the study.

Sample size calculation was done by the formula: $Z_{1-(/ 2}^{2} p$ $(1-p) / d^{2}[12]$. A total of 40 children were included in the study. This study was conducted after getting approval from the institutional ethics committee and informed consent was obtained from the parents or legal guardians. A detailed history was taken including presenting features, past history, family history, birth history, developmental milestones, and dietary history. Skin hyperpigmentation at terminal phalanges, hands, wrist, elbow, ankle, knee, and any other parts of the body was noted. Hair color was recorded. Hair changes in ITS cases were hypopigmentation, sparse, and lusterless. Pallor was looked at palpebral conjunctiva, tongue, nails, and palmer creases. Type of tremor was noted as generalized or local. Investigations including complete blood count (CBC) with blood indices (mean corpuscular volume, mean corpuscular hemoglobin [MCH], and $\mathrm{MCH}$ concentration), peripheral blood smear, and serum Vitamin B12 level were done of every case. Peripheral venous blood sample was taken in an ethylenediaminetetraacetate vial $(2 \mathrm{ml})$ for the determination of $\mathrm{CBC}$ and $3 \mathrm{ml}$ of blood was taken in a plain vial for the estimation of serum Vitamin B12 level. Serum Vitamin B12 level was estimated by electrochemiluminescence method. Serum Vitamin B12 levels in this study were defined as low $<200 \mathrm{pg} / \mathrm{ml}$ [13].

Statistical analyses were performed using the SPSS Windows, version 21. Association of different aspects of ITS with serum Vitamin B12 (cobalamin) level was calculated by Chi-square test. $\mathrm{p}<0.05$ was considered statistically significant.

\section{RESULTS}

A total of 40 children clinically diagnosed as ITS, according to the inclusion criteria, and were enrolled for the study. Maximum numbers of the children $(77.5 \%, 31)$ were in the age group of 6-12 months followed by $20 \%$ (8) in $13-18$ months and only $2.5 \%$
(1) of cases in 19-24 months age group. Among 40 children, 28 were male $(70 \%)$ and 12 were female $(30 \%)$ and male-to-female ratio was $2.3: 1$. The mean age of affected children was 10.8 months.

All patients with ITS had pallor (100\%) and skin hyperpigmentation (100\%). Psychomotor changes in the form of apathy and/or developmental regression/stasis were found in $100 \%$ of cases. Apathy was found in all cases. Developmental abnormality was found in $90 \%$ of the children. Regression of developmental milestones was found in $26(65 \%)(p<0.05)$ and $10(25 \%)$ patient had stasis of milestones. Tremor was present in $15(37.5 \%)$ cases among which $9(22.5 \%)$ had generalized tremor and rest $6(15 \%)$ patients had localized tremor. Hepatomegaly was present in $25(62.5 \%)$ of these children, whereas splenomegaly was present in only $8(20 \%)$ patients. Hypopigmented hair was found in only $12(30 \%)$ cases.

Twenty-five $(62.5 \%)$ children were exclusively breastfed. Majority of the patients 22 (71\%) of 6-12 months were exclusively breastfed. Top feeding in the form of diluted cow or goat milk was given in 8 (20\%) cases. Faulty feeding was found in 33 (82.5\%) cases (Table 1). Exclusive breastfeeding pattern was significantly associated with ITS $(\mathrm{p}<0.05)$.

Of 40 cases, $26(65 \%)$ cases presented in prodromal phase and $14(35 \%)$ presented in the tremor phase. Of 26 patients with prodromal phase, $22(84.62 \%)$ had Vitamin B12 deficiency, whereas $9(64.28 \%)$ cases of tremor phase had Vitamin B12 deficiency. Rest of the children had normal or increased Vitamin B12 level (Table 2).

Majority of the ITS children had macrocytic anemia $26(65 \%)$ followed by dimorphic anemia $10(25 \%)$. Of the remaining four cases, $2(5 \%)$ patients had microcytic and $2(5 \%)$ had normocytic anemia. Twenty-six (65\%) ITS patients had macrocytic anemia and out of them, $21(80.77 \%)$ cases were Vitamin B12 deficient also $(\mathrm{p}<0.05)$.

\section{DISCUSSION}

In the present study, all cases of ITS had pallor which was similar to the study done by Garg and Srivastava [14] and

Table 1: Feeding pattern in relation to age group

\begin{tabular}{|c|c|c|c|c|c|}
\hline $\begin{array}{l}\text { Feeding } \\
\text { pattern }\end{array}$ & $\begin{array}{c}6-12 \\
\text { months } \\
n=31(\%)\end{array}$ & $\begin{array}{c}13-18 \\
\text { months } \\
n=8(\%)\end{array}$ & $\begin{array}{c}19-24 \\
\text { months } \\
n=1(\%)\end{array}$ & $\begin{array}{c}\text { Total } \\
n=40(\%)\end{array}$ & p value \\
\hline $\mathrm{EBF}$ & $22(71)$ & $3(7.5)$ & $0(0)$ & $25(62.5)$ & 0.001 \\
\hline $\mathrm{BF}+\mathrm{TF}$ & $6(20)$ & $2(5)$ & $0(0)$ & $8(20)$ & 0.065 \\
\hline $\mathrm{BF}+\mathrm{CF}$ & $3(9)$ & $3(7.5)$ & $1(2.5)$ & $7(17.5)$ & 0.12 \\
\hline
\end{tabular}

Table 2: Serum Vitamin B12 status in relation to phase of ITS

\begin{tabular}{lcc}
\hline Phase of ITS & \multicolumn{2}{c}{ Vitamin B12 (\%) } \\
\cline { 2 - 3 } & Deficiency & Normal or increased \\
\hline Prodromal $(\mathrm{n}=26)$ & $22(84.62)$ & $4(15.38)$ \\
Tremor $(\mathrm{n}=14)$ & $9(64.28)$ & $5(35.72)$ \\
Total $(\mathrm{n}=40)$ & $31(77.5)$ & $9(22.5)$ \\
\hline
\end{tabular}

ITS: Infantile tremor syndrome 
Jadhav et al. [7]. In this study, skin hyperpigmentation, psychomotor changes and apathetic look, and developmental abnormality were reported in the majority of cases as reported in the earlier studies by Sachdev et al. [15]. Ramakumar and Pandove [4] reported hair changes in $98.1 \%$, dark skin in $88.8 \%$, and delayed milestone in $80.3 \%$ of cases as observed in our study. Significant developmental abnormality was also observed by Jadhav et al. [7] and Srikantia and Reddy [5]. Apart from the known features of ITS, significant incidence of hepatomegaly, splenomegaly, and tremor was noted as observed in other studies $[3,16,17]$.

Vitamin B12 is water-soluble vitamin required for hematopoiesis, myelination of central nervous system, and for psychomotor development. Deficiency of Vitamin B12 can occur due to poor dietary intake in children with faulty feeding, prolong exclusive breastfeeding by Vitamin B12-deficient mother. Vitamin B12 deficiency impairs DNA maturation during hematopoiesis which leads to ineffective erythropoiesis and macrocytic anemia. Vitamin B12 deficiency also impairs psychomotor development and myelination of nervous system which leads to developmental abnormality and tremors.

As malnutrition is commonly associated with ITS, fatty infiltration of liver can lead to hepatomegaly. Lower respiratory tract infection and malaria can present with splenomegaly. In this study, majority of children were on faulty feeding in the form of prolonged exclusive breastfeeding without proper complementary feeding and diluted cow or goat milk. In such children, there were deficiencies of Vitamin B12, multiple macro- and micro-nutrients which lead to manifestations in the form of anemia, skin hyperpigmentation, tremor, and psychomotor changes. Similar result was observed in the previous studies $[3,18,19]$.

In the present study, $31(77.5 \%)$ children had low serum Vitamin B12 level. About $84.62 \%$ of cases of prodromal stage were found Vitamin B12 deficient, while $64.28 \%$ of cases of tremor stage were found Vitamin B12 deficient. Similarly, Jadhav et al. and Srikantia and Reddy reported low serum Vitamin B12 level in $100 \%$ and $87.5 \%$ of patients of ITS, respectively [5,7]. The most common type of anemia diagnosed by peripheral smear was macrocytic $26(65 \%)$ followed by dimorphic $10(25 \%)$ in our study. Of 26 cases of macrocytic anemia, $21(80.77 \%)$ cases had Vitamin B12 deficiency.

Vitamin B12 deficiency impairs DNA maturation during hematopoiesis which leads to ineffective erythropoiesis and large size red blood cell production seen as macrocyte in peripheral blood smear. Similar results were shown by other studies also $[3,15]$. Of 40 cases of ITS, 9 (22.5\%) children had normal or increased Vitamin B12 level in our study. Various possible causative factors other than Vitamin B12 deficiency have been postulated such as malnutrition, vitamin, and mineral deficiency (e.g. $\mathrm{Mg}$ and $\mathrm{Zn}$ ), infections, toxins, degenerative brain disease, and enzyme defects (tyrosine) [20]. The study was limited by its small sample size. Other investigations suggestive of Vitamin B12 deficiency such as the urinary methylmalonic acid (MMA) level, serum homocysteine levels, and serum MMA were not done in our study.

\section{CONCLUSION}

We conclude that all children of ITS presented with anemia, skin hyperpigmentation, psychomotor changes, apathetic look, and developmental abnormality. Majority of children were on faulty feeding in the form of prolonged exclusive breastfeeding without proper complementary feeding. Vitamin B12 deficiency was present in a significant number of patients with ITS. A significant number of ITS patients had macrocytic anemia and out of them, most of the cases were Vitamin B12 deficient also. This study provides a unique hospital-based biochemically defined estimate of Vitamin B12 deficiency as a strong causal relationship with ITS.

\section{AUTHORS' CONTRIBUTIONS}

Data collection was done by Dr. Dhaval Bhatt and Dr. Ishwar Lal Meena. Analysis and manuscript preparation were done by Dr. Gopikishan Sharma and Dr. Jitendra Kumar Jain. All research works had been done under the guidance of Dr. A. L. Bairwa.

\section{REFERENCES}

1. Kalra V. Central nervous system. In: Paul VK, Bagga A, Sinha A, editors. Ghai Essential Pediatrics. $8^{\text {th }}$ ed. New Delhi: CBS Publisher and Distributors; 2013. p. 580-1.

2. Gupte S, Pal M, Gupta SK, Sangra KR. Infantile tremor syndrome (ITS). In: Gupte S, editor. Short Text Book of Pediatrics. $12^{\text {th }}$ ed. New Delhi: Jaypee Brothers; 2016. p. 541.

3. Agarwal SP, Katiyar GP. Infantile tremor syndrome. Pediatr Clin India 1972; 7:203-8.

4. Ramakumar L, Pandove SP. Infantile tremor syndrome. Indian J Pediatr 1975;42:215-25.

5. Srikantia SG, Reddy V. Megaloblastic anaemia of infancy and Vitamin B12. Br J Haematol 1967;13:949-53.

6. Holla RG, Prasad AN. Infantile tremor syndrome. Med J Armed Forces India 2010;66:186-7.

7. Jadhav M, Webb JK, Vaishnava S, Baker SJ. Vitamin B12 deficiency in Indian infants. A clinical syndrome. Lancet 1962;2:903-7.

8. Avci Z, Turul T, Aysun S, Unal I. Involuntary movements and magnetic resonance imaging findings in infantile cobalamine (Vitamin B12) deficiency. Pediatrics 2003;112:684-6.

9. Vora RM, Tullu MS, Bartakke SP, Kamat JR. Infantile tremor syndrome and zinc deficiency. Indian J Med Sci 2002;56:69-72.

10. Ratageri VH, Shepur TA, Patil MM, Hakeem MA. Scurvy in infantile tremor syndrome. Indian J Pediatr 2005;72:883-4.

11. Sirolia V, Arya S. Study of clinical profile and estimation of Vitamin B12 level in infantile and pre-infantile tremor syndrome. J Evol Med Dent Sci 2014;3:10134-7.

12. Kasiulevicius V, Sapoka V, Filipaviciute R. Sample size calculation in epidemiological studies. Gerontology 2006;7:225-31.

13. Langan RC, Zawistoski KJ. Update on Vitamin B12 deficiency. Am Fam Physician 2011;83:1425-30.

14. Garg BK, Srivastava JR. Infantile tremor syndrome. Indian J Pediatr 1969;36:213-8

15. Sachdev KK, Manchanda SS, Lal H. The syndrome of tremors, mental regression and anaemia in infants and young children: A study of 102 cases. Indian Pediatr 1965;2:239-51.

16. Bajpai PC, Tandon PN, Misra PK, Sharma NL. Infantile tremor syndrome. Acta Neurol Scand 1965;41:473-6. 
17. WHO/UNICEF/UNU. Iron Deficiency Anemia Assessment Prevention and Control. A Guide for Programme Managers. Geneva, Switzerland: WHO; 2001.

18. Amin NM, Zeki JM. Infantile tremor syndrome in Iraqi Kurdistan. Indian J Pediatr 2005;72:839-42.

19. Kaul KK. Syndrome of tremors in Indian infants. J Indian Med Assoc 1972;59:473-5.

20. Kalra V. Infantile tremor syndrome. In: Ghai OP, editor. Essential Pediatrics. $7^{\text {th }}$ ed. New Delhi: CBS Publishers and Distributions Pvt. Ltd.; 2009. p. 558-9.
Funding: None; Conflict of Interest: None Stated.

How to cite this article: Jain JK, Sharma G, Bhatt D, Bairwa AL, Meena IL. Study of clinical profile of infantile tremor syndrome and its correlation with serum Vitamin B12 level. Indian J Child Health. 2019; 6(7):345-348.

Doi: 10.32677/IJCH.2019.v06.i07.004 